\title{
Hypersensitivity to molybdenum as a possible trigger of ANA-negative systemic lupus erythematosus
}

\author{
Martin Federmann, Beat Morell, Galleon Graetz, Myriam Wyss, Peter Elsner, \\ Regula von Thiessen, Brunello Wüthrich, Dieter Grob
}

\begin{abstract}
After implantation of two metal plates a 24 year old woman developed fever of unknown origin and successively more symptoms of an ANA-negative systemic lupus erythematosus (SLE). These symptoms resolved after removal of the plates and recurrred during patch testing of the metal components, which showed a reaction to molybdenum. A lymphocyte transformation test indicated a delayedtype hypersensitivity to molybdenum. Subsequent progressive flare ups of SLE appeared without molybdenum reexposure. This is the first report suggesting the existence of a hypersensitivity to molybdenum, which may act as another environmental trigger for SLE.
\end{abstract}

(Ann Rheum Dis 1994; 53: 403-405)

In the pathogenesis of systemic lupus erythematosus (SLE) both genetic predisposition and environmental triggers are likely to play an important role with differing factors for different individuals. ${ }^{1}$ These factors can induce a loss of self-tolerance, a polyclonal B-cell activation and/or an antigen-driven immune response, which subsequently give rise to the production of characteristic autoantibodies and lead to the highly variable clinical manifestations of the disease. ${ }^{12}$ Potential environmental triggers are heterogenous and seem to act by distinct mechanisms. According to Krieg ${ }^{1}$ ultra-violet light, certain drugs like procainamide, and hair dyes, possibly some chemicals (such as hydrazine), certain foods, stress, and infections, and other agents (such as tobacco smoke) are thought to be implicated in the induction or activation of SLE.

We describe a patient showing evidence that a hypersensitivity to molybdenum induced manifestations of an ANA-negative systemic lupus erythematosus.

\section{Case report}

A 24 year old woman had a history of posttraumatic splenectomy at the age of five years and a contact allergy to phenylmercurialborate. She had not used drugs known to be involved in lupus-induction or hair dyes. Following a whiplash injury in April 1988 she developed a severe chronic cervical syndrome. Four weeks after a successful event-free stabilisation-test with external cervical fixation consisting of a chromium-nickel-alloy with a content of $2 \cdot 8 \%$ molybdenum, two metal plates of the same alloy were implanted for posterior fusion of segments C6-Th1, on 17 October 1990. Fifteen hours later she experienced a persistent remittent fever of up to $39.5 \mathrm{C}$. No infectious, drug-related or other cause could be found. Sedimentation rate, CRP, complements factors (C-3c, C-4, B) and anti-RNP were normal. Antinuclear antibodies (ANA) (Immunofluorescence(IF) tests using rat liver sections and Hep-2 tissue culture cells), anti-dsDNA antibodies (enzyme linked immunosorbent assay using recombinant antigen and IF-test using Crithidia luciliae) and rheumatoid factor tested consistently negative throughout the follow up. The fever responded to ibuprofen. The patient was discharged in good condition on 6 December 1990. A review of previous reports and other investigations revealed that metal implants were not known to cause long standing non-infectious postoperative fever.

In January 1991 the patient complained temporarily about painless symmetrical morning swellings of her proximal interphalangeal and ankle joints. After three months of persistent fever she was readmitted to the hospital for further examination on 6 February 1991. A few shallow oral ulcerations had appeared. Diagnostic procedures including biopsies of bone marrow, lymph nodes and liver revealed no explanation.

In March 1991 skin tests with the metal alloy were performed. Scratch testing was negative after 15 minutes, but showed a red and infiltrated reaction after six hours that proceeded to an erosion, which persisted for six weeks. Patch testing was positive with an erythematous infiltration after 48 hours, followed by an erosion at 72 hours. Six controls were negative. Two weeks later multiple oral ulcers developed followed by a massive patchy frontoparietally accentuated alopecia. Laboratory evaluation showed normochromic normocytic anaemia and slightly elevated anti-Ro/SS-A antibodies (303 U/ml; normal 0-200), tests for anti-La/ SS-B-, anti-Sm- and anti-RNP antibodies were within the normal range, values for uric acid and serum copper were normal. After the removal of the cervical metal plates on 5 April 1991 the fever subsided within one day. There was a short episode of cramping abdominal

23 February 1994

Abteilung, Spital
} 
pain that followed, and endoscopy showed nonspecific erosive gastritis and colitis. The histopathology of the peri-prosthetic tissue showed a chronic inflammation with predominating T-lymphocytes.

The patient improved until patch testing was carried out with the pure components of the metal alloy on 2 July 1991, which resulted in a similar reaction to that of March 1991 exclusively to molybdenum. The biopsy showed an eczematous reaction with parakeratosis, acanthosis and a lymphocytic and histiocytic dermal infiltrate. Subsequently fever, sweats, oral ulcers, alopecia and signs of gastroenteritis appeared successively within two weeks and resolved spontaneously within three. A similar episode including a slight papular rash of the face and neck developed after dental treatment on 5 September 1991, when molybdenum-containing materials were used.

In November 1991 another similar flare up took place, for the first time without any apparent molybdenum exposure apart from food intake. A short course of prednisone was given. Leucopenia $(3400 / \mathrm{mm} 3)$ was present. Complement factors C-3 $(0.48 \mathrm{~g} / \mathrm{l}$; normal $0 \cdot 50-1 \cdot 20)$ and $\mathrm{C}-4 \quad(0 \cdot 11 \mathrm{~g} / \mathrm{l} ;$ normal $0 \cdot 14-0 \cdot 45)$ were reduced. Antibody testing revealed elevated titres of anti-Ro/SS-A (985

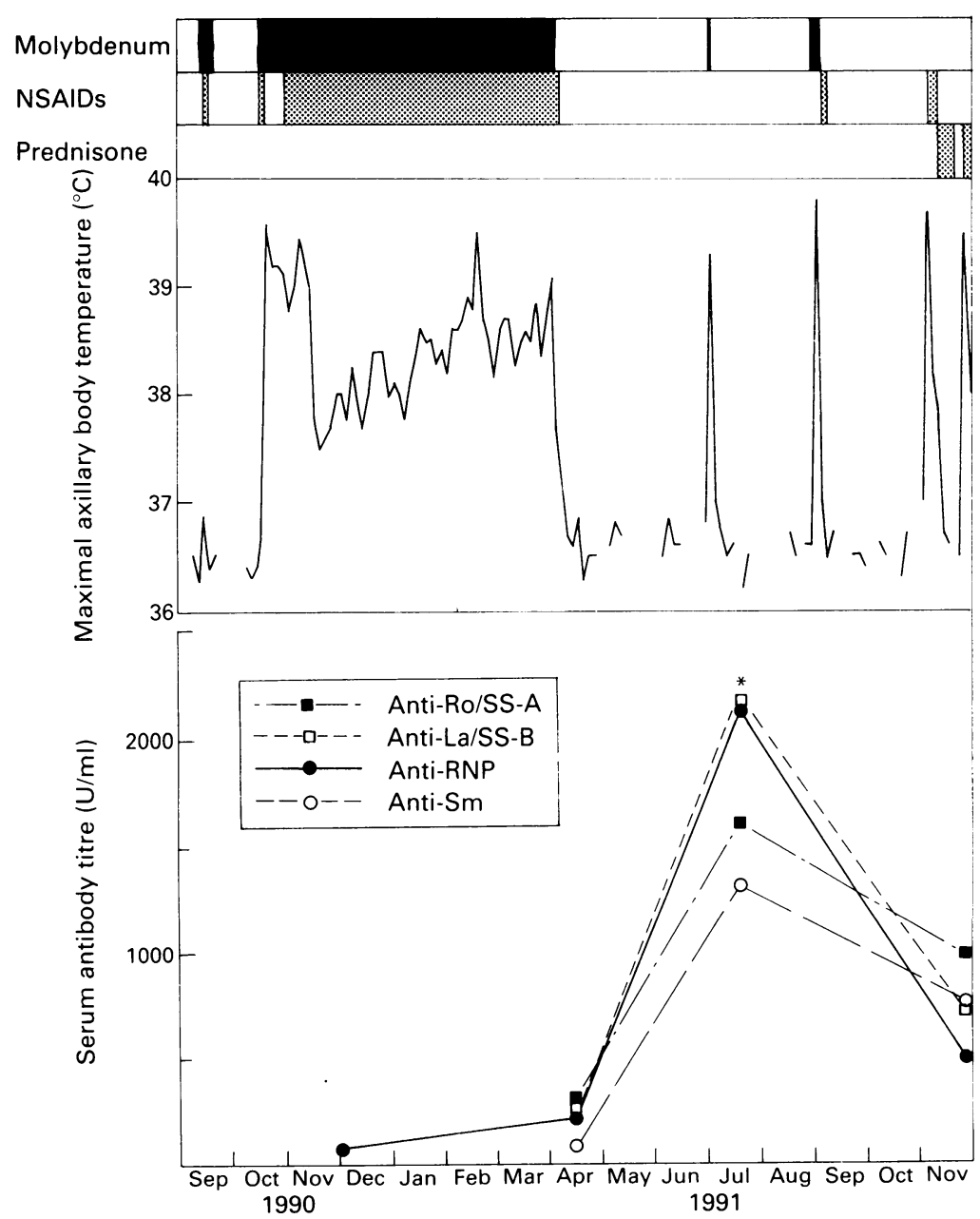

Maximal axillary body temperature and serum titres of the characteristic antibodies in relation to molybdenum exposure and therapy with nonsteroidal anti-inflammatory drugs and prednisone, NSAIDs = nonsteroidal anti-inflammatory drugs; $\operatorname{solid}$ bar $(\square$, 圈) = exposure or therapy present, white bar ( $\square$ ) exposure or therapy not present.

$\star$ The values of Fuly 1991 were measured retrospectively.
$\mathrm{U} / \mathrm{ml})$, anti-La/SS-B (795 U/ml; normal 0-300), anti-Sm (759 U/ml; normal 0-200) and anti-RNP (489 U/ml; normal 0-300); the presence of anti-Ro/SS-A, anti-Sm and antiRNP, but not of anti-La/SS-B, was later confirmed by immunodiffusion (ENA Ouchterlony test). A lymphocyte transformation test (during prednisone at $10 \mathrm{mg} / \mathrm{d}$ ) indicated a hypersensitivity to molybdenum (stimulation index 4.1 at $10 \mathrm{ppm} ; 2$ healthy controls: 1.94 and $2 \cdot 1$ ).

Afterwards flare ups recurred despite treatment with increasing doses of prednisone, and until recently cyclophosphamide. Additional clinical features included: headache, Raynaud's phenomenon, painful swelling of the right wrist, repeated pancytopenia with lymphopenia, neutropenia, Coombs positive autoimmune haemolytic anaemia and autoimmune thrombocytopenia. Signs of Sjögren's syndrome did not occur (Schirmer's test was normal). Lupus band test showed slight granular IgG- and IgM-deposits along the basement membrane zone; LE cell test and VDRL were negative. The already elevated antibody titres increased, furthermore antibodies to histones, cardiolipin-IgG, phosphatidylserine, monosialoganglioside GM1 and disialoganglioside GD1 were detected. A positive direct Coombs test with positive anticomplement; cryoglobulins and plateletassociated IgG were demonstrated. Complement factor $\mathrm{C} 2$ and total haemolytic complement $(\mathrm{CH} 50)$ were normal. The study of the major histocompatibility complex antigens revealed the following phenotypes: A2, A3, B12(44), B37, DR1, DRw11(5), DRw52, DQw1 and DQw3.

\section{Discussion}

The clinical features of this patient are consistent with the characteristics of an ANAnegative SLE: ${ }^{3}$ she fulfilled four American Rheumatism Association (ARA) criteria for classification of $\mathrm{SLE}^{4}$ (oral ulcers, arthritis, leucopenia, anti-Sm antibodies), and developed several additional symptoms of SLE, such as, Coombs positive autoimmune haemolytic anaemia and autoimmune thrombocytopenia, while ANA tested constantly negative. The term 'ANA-negative' refers only to a negative result with the IF-ANA test. This is significant because the test is generally employed in screening for SLE. The ANAnegative subset accounted for $4 \%$ of SLE cases in a recent large European SLE series, where IF-ANA tests using both human Hep-2 cells and rodent tissue sections as substrates were used as in this case. ${ }^{5}$ With other test methods antibodies of the ANA-group, especially AntiRo/SS-A, are often detectable in ANA-negative patients. ${ }^{3}$ Anti-Sm is seldom found in this setting. In that study four of 37 ANA negative cases were anti-SM positive ( $R$ Cervera on behalf of the European Working Party on Systemic Lupus Erythematosus: personal communication). The simultaneous detection of anti- Ro/SS-A, anti-Sm and anti-RNP in our case appears to be very rare. 
The clinical course (figure) provides evidence for an environmental trigger for lupus: there is a striking association between the start and duration of persistent fever and the presence of implanted metal plates, which could later be ascribed to the molybdenum content of the alloy. Similarly the subsequent two flare ups showed a close temporal relation to molybdenum contact. All the symptoms recurred later as part of the increasing lupus syndrome without apparent molybdenum exposure apart from food intake. The characteristic antibodies increased much later than the onset of the fever. For anti-Sm antibodies a correlation of the antibody titre with the disease activity of SLE has been demonstrated. ${ }^{6}$ Skin prick test reactions, histology, previous transcutaneous uneventful exposure to molybdenum but with the possibility of sensitisation, the reactions to the subsequent re-exposures, and the positive lymphocyte transformation test indicated a delayed-type hyper-sensensitivity to molybdenum. Toxic reactions to the essential trace element molybdenum are known, and include a gout-like syndrome with hyperuricaemia and low blood copper levels. ${ }^{7}$ None of these symptoms was present in our patient. To our knowledge, this is the first report suggesting that molybdenum may be a further environmental trigger for the induction of SLE and for a hypersensitivity to molybdenum. The cause of this association remains obscure.

As a possible indication of a genetic predisposition for SLE the HLA phenotypes DQw1 and DRw52 are present. Both have been found in excess in white groups with SLE. ${ }^{8}$

Our patient had had a splenectomy in childhood but the effect of a missing spleen in the development of lupus is not clear. There are a few reports, presenting evidence for the worsening of lupus ${ }^{9}$ or other autoimmune disease ${ }^{10}$ after splenectomy in adults.
The presenting feature was fever of unknown origin. Systemic lupus erythematosus, formerly an important cause of unexplained fever, has disappeared almost completely from the spectrum of responsible entities, since diagnosis has become easier with the availability of IF-ANA testing. ${ }^{11}$ The reported case demonstrates that ANA-negative SLE can still be a puzzling reason for fever of unknown origin unless repeated testing of single nuclear antibodies, especially of anti-Ro/ SS-A, is performed.

We thank Professor Beat Michel for his helpful comments, D Helen I Joller-Jemelka, Professor Arthur von Felten and Professor Werner J Pichler for their expert laboratory testing, and Professor Ortrun Pohler for providing the components of the metal alloy.

1 Steinberg A D, Gourley M F, Klinman D M, Tsokos G C, Scott D E, Krieg A M. Systemic lupus erythematosus. Ann Intern Med 1991; 115: 548-59.

2 Boitard C. B-cell responses in autoimmune diseases. Curr Opin Immunol 1992; 4: 741-7.

3 Maddison P J, Provost T T, Reichlin M. Serological findings in patient with "ANA-negative" systemic lupus erythematosus. Medicine (Baltimore) 1981; 60: 87-94.

4 Tan E M, Cohen A S, Fries J F, et al. The 1982 revised criteria for the classification of systemic lupus erythematosus. Arthritis Rheum 1982; 25: 1271-7.

5 Cervera R, Khamashta M A, Font J, et al and the European Working Party on Systemic Lupus Erythematosus. Systemic lupus erythematosus: clinical and immunologic patterns of disease expression in a cohort of 1,000 patients. Medicine (Baltimore) 1993; 72: 113-24.

6 Barada F C, Andrews B S, Davis I V J S, Taylor R P. Antibodies to $\mathrm{Sm}$ in patients with systemic lupus erythematosus. Correlation of $\mathrm{Sm}$ antibody titers with disease activity and other laboratory paremeters. Arthritis disease activity and other labo

7 Rajagopalan K V. Molybdenum: An essential trace element in human nutrition. Ann Rev Nutr 1988; 8: 401-27.

8 Hochberg M C, Boyd R E, Ahearn J M, et al. Systemic lupus erythematosus: A review of clinico-laboratory features and immunogenetic markers in 150 patients with emphasis on demographic subsets. Medicine (Baltimore) 1985; 65: 285-95.

9 Dameshek W, Reeves W H. Exacerbation of lupus erythematosus following splenectomy in "idiopathic" throbocytopenic purpura and autoimmune hemolytic anemia. Am $\mathcal{F}$ Med 1956; 21: 560-6.

10 Cales P, Howaizi M, Vinel J P, Pascal J P. Could splenectomy worsen autoimune manifestations? (Letter). f Intern Med 1990; 228: 411

11 Larson E B, Featherstone H J, Petersdorf R G. Fever of undetermined origin: Diagnosis and follow-up of 105 cases, 1970-1980. Medicine (Baltimore) 1982; 61: 269-92. 